\title{
Therapeutic efficacy and safety of combined BRAF and MEK inhibition in patients with malignant melanoma: a meta-analysis
}

This article was published in the following Dove Press journal:

OncoTargets and Therapy

I 3 November 2017

Number of times this article has been viewed

\author{
Peng Chen ${ }^{1, *}$ \\ Fuchao Chen ${ }^{2, *}$ \\ Benhong Zhou' ${ }^{1,3}$ \\ 'Department of Pharmacy, Renmin \\ Hospital of Wuhan University, Wuhan, \\ Hubei, ${ }^{2}$ Department of Pharmacy, \\ Dongfeng Hospital, Hubei University \\ of Medicine, Shiyan, Hubei, ${ }^{3}$ School \\ of Pharmaceutical Sciences, Wuhan \\ University, Wuhan, Hubei, People's \\ Republic of China
}

*These authors contributed equally to this work
Correspondence: Benhong Zhou Department of Pharmacy, Renmin Hospital of Wuhan University, Zhang Zhi Dong Road, Wuhan District, Hubei, 430060, People's Republic of China Tel +86 I5 33589843 I

Email benhongzh@whu.edu.cn
Background: Recent clinical studies have shown that initial therapy with combined $B R A F$ and mitogen-activated extracellular signal-regulated kinase (MEK) inhibition is more effective in metastatic melanoma than single-agent $B R A F$ inhibitors. However, the response rates with single-agent $B R A F$ are low. Thus, the objective of this study was to conduct a meta-analysis of randomized controlled trials to compare the efficacy and adverse events risk between monotherapy and combination therapy.

Materials and methods: Searches were made in PubMed and EMBASE electronic databases and conference abstracts published by the American Society of Clinical Oncology from 2000 to 2017. Outcomes included overall response, progression-free survival, and overall survival, as well as the incidence rate of adverse events.

Results: Eight trials comprising 2,664 patients were included in the meta-analysis. Patients with combined therapies showed superior results compared to those with $B R A F$ inhibitors alone for the following: overall response rate (combined relative risk $[R R]=1.34,95 \%$ confidence interval [95\% CI]: 1.24-1.45, $P<0.00001$ ), progression-free survival (combined hazards ratio $[\mathrm{HR}]=0.58,95 \% \mathrm{CI}: 0.52-0.64, P<0.00001$ ), and overall survival rate (combined HR $=0.70$, 95\% CI: $0.62-0.80, P<0.00001)$. Patients with combination therapies had higher incidence of adverse events including pyrexia (combined $\mathrm{RR}=2.00,95 \% \mathrm{CI}$ : $1.40-2.84$ ), nausea (combined $\mathrm{RR}=1.41,95 \% \mathrm{CI}: 1.03-1.94$ ), diarrhea (combined $\mathrm{RR}=1.50,95 \% \mathrm{CI}: 1.08-2.06$ ), and vomiting (combined RR $=1.87,95 \%$ CI: 01.52-2.31) compared to those with $B R A F$ inhibitors alone.

Conclusion: These data suggested that the combined $B R A F$ and MEK inhibition was associated with a significant improvement in overall response, progression-free survival, and overall survival, but increased the incidence of adverse events among the patients with BRAF V600mutated metastatic melanoma. Further large-scale, high-quality, placebo-controlled, double-blind trials are needed to confirm this conclusion.

Keywords: efficacy, safety, melanoma, meta-analysis, $B R A F$ inhibition, MEK inhibition

\section{Introduction}

The global incidence of melanoma is increasing, with about 200,000 new cases and about 65,000 melanoma-associated deaths every year. ${ }^{1}$ Although metastatic melanoma typically has been associated with poor prognosis, with low survival averaged by $15 \%-60 \%$ in patients, the therapeutic options recently changed substanti-ally. ${ }^{2}$ After the approval of $B R A F$ inhibitor such as vemurafenib or dabrafenib by the United States Food and Drug Administration (US FDA) in 2011, there has been a significant improvement in the response rates, progression-free survival (PFS), and overall survival (OS), in patients with metastatic melanoma with $B R A F$ V600E or V600K mutations, 
compared with chemotherapy. ${ }^{3}$ However, the mitogenactivated protein kinase (MAPK) pathway was frequently reactivated, thereby causing acquired resistance to $B R A F$ inhibitors. In addition, the use of $B R A F$ inhibitors may result in the development of secondary skin tumors, originating from a paradoxical activation of the MAPK pathway in cells without a $B R A F$ mutation. ${ }^{4}$ In recent years, clinical benefits of combining a $B R A F$ inhibitor with a mitogen-activated extracellular signal-regulated kinase (MEK) inhibitor were initially shown in several randomized trials, which found that it can address not only the limitations of single-agent $B R A F$ inhibitors, but also improve the PFS and OS than with $B R A F$ inhibitors alone. ${ }^{5,6}$

Although combined use of $B R A F$ and MEK inhibitors is well tolerated by many patients, it is not devoid of side effects. Several clinical trials reported that diarrhea, anorexia, nausea, and vomiting are common adverse events frequently associated with the use of a combination of $B R A F$ and MEK inhibitors in daily clinical practice, thus requiring early and appropriate managements to avoid unnecessary dose reductions and transitory or definitive treatment discontinuations. ${ }^{7}$ Therefore, there is a need to master the characteristic features, incidence, and relative risk (RR) of significant adverse events to take adequate prevention and intervention as early as possible. ${ }^{8}$ Hence, in this study, we have performed a meta-analysis to assess the therapeutic efficacy and safety of combined $B R A F$ and MEK inhibition in patients with malignant melanoma and to provide treatment recommendations for these symptoms.

\section{Materials and methods}

\section{Search strategy}

The PubMed, EMBASE, and Cochrane Library electronic databases were searched for articles published from January 2000 to January 2017 using keywords " $B R A F$ inhibition", "MEK inhibition", "Dabrafenib”, "Trametinib”, "Vemurafenib", "Cobimetinib", "Melanoma", "Val600", and " $B R A F$-mutant". Additionally, the abstracts presented at major meetings of the American Society of Clinical Oncology (ASCO), the European Society for Medical Oncology, and the World Lung Cancer Conference were manually searched. Finally, full publications (not abstracts) from the Web of Science database were also searched to ensure that there were no additional studies.

\section{Study selection}

Studies that met the following criteria were included in the study: 1) studies of prospective Phase II and Phase III clinical trials and expanded-access (ie, outside clinical trials) programs, 2) studies that clinically investigated patients with melanoma or metastatic melanoma and those that studied the combinatorial effect of the participants assigned to $B R A F$ and MEK inhibitors, and 3) studies that analyzed efficiency measures including overall response rate (ORR), PFS, and OS, as well as adverse events. The exclusion criteria were as follows: 1) investigations in patients of original studies that were unrelated to the study drug and 2) original studies that met the inclusion criterion (1) but required information such as overall response, PFS, and OS, as well as adverse events, etc, were not available.

\section{Data extraction and quality assessment}

We extracted information such as the first author, number of patients enrolled in the study, treatment information and characteristics of the participants to understand the baseline of all the included studies. To determine the validity of the selected studies, a modified Jadad scale was used to assess the quality of the included randomized studies. The scores of high-quality studies ranged from 4 to 8 , whereas that of low-quality studies from 0 to 3. For non-randomized studies, the Newcastle-Ottawa Quality Assessment Scale was used. Each study was graded as either of low quality (0-5) or high quality (6-9). Any disagreements were resolved by discussion among our investigators or by referring to the original publication.

\section{Definition of main outcomes}

Complete response was defined as disappearance of all symptoms and signs of all measurable disease for at least 4 weeks and no appearance of any new lesions during that time. Partial response was defined as a 50\% reduction in the sum of the products of the perpendicular diameters of all measurable lesions for at least 4 weeks and no new lesions or no enlargement in existing lesions during that time. Overall response included complete response and partial response. PFS was defined as one type of measurement that can be used in a clinical study or trial to help determine whether a new treatment is effective or not. It refers to the probability that a patient will remain alive, without the disease getting worse. We defined the OS as the time from the date of randomization to the date of death from any cause, or the date of last follow-up of a living patient. As for the safety outcomes, we referred to the trial authors' definitions. We collected data about five frequent toxicity events (pyrexia, nausea, diarrhea, vomiting, and arthralgia) to enter into the meta-analysis. 


\section{Statistical analysis}

Meta-analyses were carried out using software Review Manager Version 5.3 and R 2.13.2 Meta package. The pooled RR (risk ratio) and HR (hazard ratio) estimate and 95\% confidence intervals (CIs), stratified by study setting and gender, were conducted using a random- or fixed-effect model, with between-study heterogeneity assessed using $I^{2}$ statistic. If $I^{2}$ was $\geq 50 \%$, then the random-effect model was used, and if not, then the fixed-effect model was used. Statistical significance was defined as a $P$-value $<0.05$. All 95\% CIs were two-sided. Finally, the publication bias were quantitatively tested by conducting Egger's test.

\section{Results}

\section{Search results}

\section{Study selection}

Of the studies initially identified, we excluded reports that did not meet the inclusion criteria after first screening the study titles and abstracts. Finally, eight studies ${ }^{9-16}$ were ultimately included in the meta-analysis. Figure 1 illustrates how the eight studies were obtained from the literature search. The eight selected studies (including six randomized controlled trials), comprising a total of 2,664 participants, were published between 2012 and 2017. In all studies (including three Phase I/II studies ${ }^{10,12}$ and five Phase III studies ${ }^{9,11,13,14}$ ), the starting dose and schedule of vemurafenib (dabrafenib) and cobimetinib (trametinib) was based on US FDA guidelines (960 mg vemurafenib, bid; 60 or $80 \mathrm{mg}$ cobimetinib, qd; $150 \mathrm{mg}$ dabrafenib, bid; $2 \mathrm{mg}$ trametinib qd). The main characteristics and qualities of included trials are listed in Table 1. Jadad scores of each of the eight studies included in the meta-analysis are also listed in Table 1; the mean score was 4.25 (range, 3-6), which indicates that the overall quality of the study was fair.

\section{Effectiveness}

In our meta-analysis, four comparisons were used to analyze ORR and six comparisons to analyze PFS and OS.

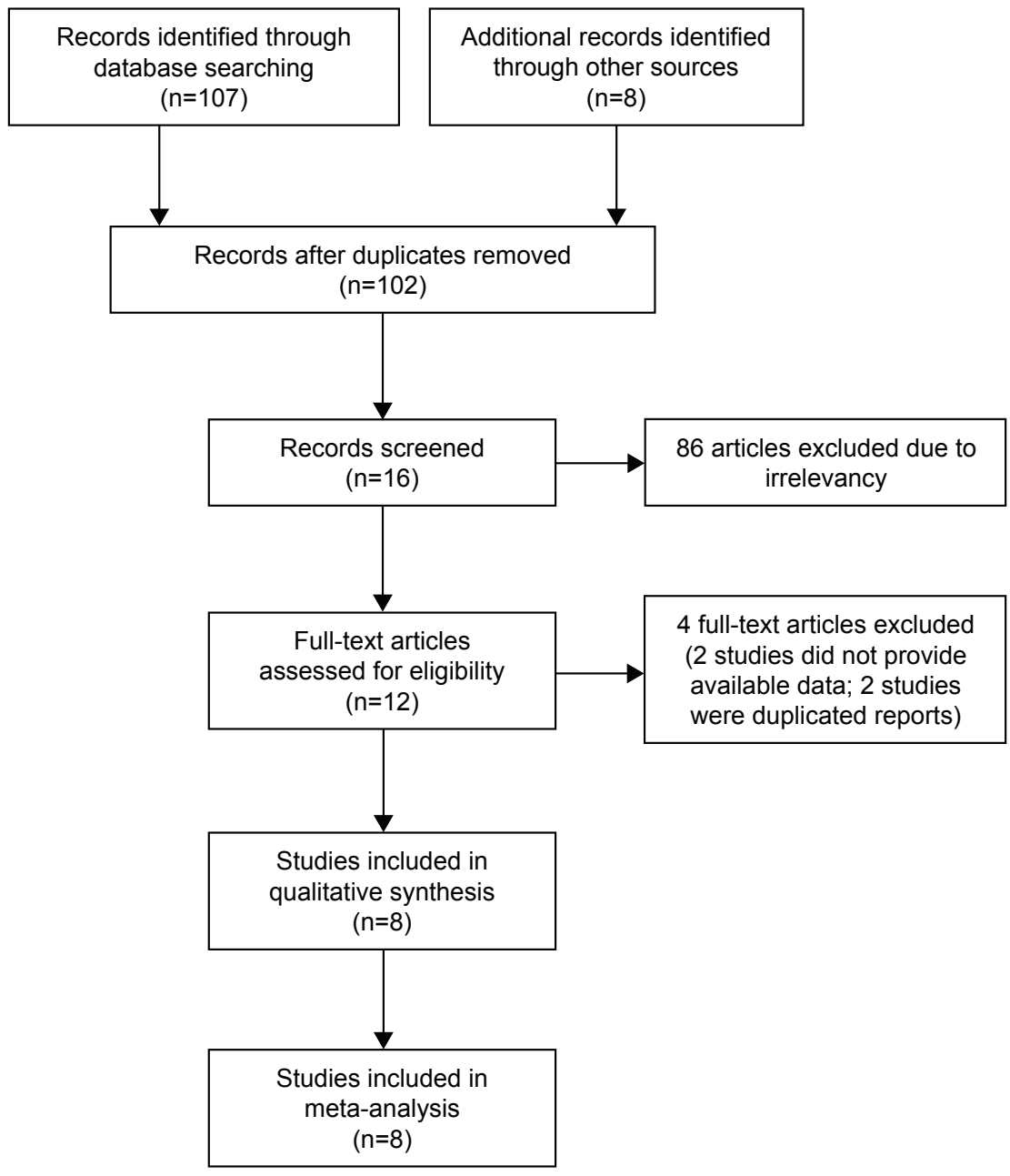

Figure I Flow diagram of the study selection process. 
Table I Summary of the characteristics of the studies included in the meta-analysis

\begin{tabular}{|c|c|c|c|c|c|}
\hline Reference (year) & Trial design & Patients (N) & $\begin{array}{l}\text { Age, years, } \\
\text { median (range) }\end{array}$ & Treatment regimen & $\begin{array}{l}\text { Quality } \\
\text { score }\end{array}$ \\
\hline \multirow[t]{3}{*}{ Long et al $(2017)^{9}$} & Phase III & 423 & & & \\
\hline & $\mathrm{D}+\mathrm{T}$ vs $\mathrm{D}$ & $211(\mathrm{D}+\mathrm{T}) / 212(\mathrm{D})$ & $55.5(23-89)$ & $\mathrm{D}(150 \mathrm{mg}, \mathrm{bid})+\mathrm{T}(2 \mathrm{mg}, \mathrm{qd})$ & 5 \\
\hline & & & & vs D (I50 mg, bid) & \\
\hline \multirow[t]{2}{*}{ Johnson et al $(2014)^{10}$} & Phase I/II & 45 & & & \\
\hline & $\mathrm{D}+\mathrm{T}$ & $45(\mathrm{D}+\mathrm{T})$ & $51.0(18-82)$ & $\mathrm{D}(150 \mathrm{mg}, \mathrm{bid})+\mathrm{T}(2 \mathrm{mg}, \mathrm{qd})$ & 4 \\
\hline \multirow[t]{3}{*}{ Long et al (2015)"' } & Phase III & 423 & & & \\
\hline & $\mathrm{D}+\mathrm{T}$ vs $\mathrm{D}$ & $211(\mathrm{D}+\mathrm{T}) / 212(\mathrm{D})$ & $56.5(22-86)$ & $\mathrm{D}(150 \mathrm{mg}, \mathrm{bid})+\mathrm{T}(2 \mathrm{mg}, \mathrm{qd})$ & 5 \\
\hline & & & & vs D (I50 mg, bid) & \\
\hline \multirow[t]{3}{*}{ Larkin et al $(2014)^{12}$} & Phase III & 495 & & & \\
\hline & $\mathrm{V}+\mathrm{C}$ vs V & $247(\mathrm{~V}+\mathrm{C}) / 248(\mathrm{~V})$ & $55.0(23-88)$ & $\mathrm{V}(960 \mathrm{mg}, \mathrm{bid})+\mathrm{C}(60 \mathrm{mg}, \mathrm{qd})$ & 4 \\
\hline & & & & vs $\vee(960$ mg, bid $)$ & \\
\hline \multirow[t]{3}{*}{ Schadendorf et al $(2015)^{13}$} & Phase III & 423 & & & \\
\hline & $\mathrm{D}+\mathrm{T}$ vs $\mathrm{D}$ & $211(\mathrm{D}+\mathrm{T}) / 212(\mathrm{D})$ & $55.0(25-85)$ & $\mathrm{D}(150 \mathrm{mg}, \mathrm{bid})+\mathrm{T}(2 \mathrm{mg}, \mathrm{qd})$ & 3 \\
\hline & & & & vs D (I50 mg, bid) & \\
\hline \multirow[t]{3}{*}{ Robert et al $(2015)^{14}$} & Phase III & 704 & & & \\
\hline & $\mathrm{D}+\mathrm{T}$ vs $\mathrm{V}$ & $352(\mathrm{D}+\mathrm{T}) / 352(\mathrm{~V})$ & $55.0(|8-9|)$ & $\mathrm{D}(150 \mathrm{mg}, \mathrm{bid})+\mathrm{T}(2 \mathrm{mg}, \mathrm{qd})$ & 4 \\
\hline & & & & vs $\mathrm{V}(960 \mathrm{mg}, \mathrm{bid})$ & \\
\hline \multirow[t]{2}{*}{ Corcoran et al $(2015)^{15}$} & Phase I/II & 43 & & & \\
\hline & $\mathrm{D}+\mathrm{T}$ & $43(\mathrm{D}+\mathrm{T})$ & $55.0(42-69)$ & $\mathrm{D}(\mathrm{I} 50 \mathrm{mg}, \mathrm{bid})+\mathrm{T}(2 \mathrm{mg}, \mathrm{gd})$ & 3 \\
\hline \multirow[t]{3}{*}{ Flaherty et al $(2012)^{16}$} & Phase I/II & 423 & & & \\
\hline & $\mathrm{D}+\mathrm{T}$ vs $\mathrm{D}$ & $211(\mathrm{D}+\mathrm{T}) / 212(\mathrm{D})$ & $58(27-79)$ & $\mathrm{D}(150 \mathrm{mg}, \mathrm{bid})+\mathrm{T}(2 \mathrm{mg}, \mathrm{qd})$ & 6 \\
\hline & & & & vs $D(150 \mathrm{mg}, \mathrm{bid})$ & \\
\hline
\end{tabular}

Abbreviations: bid, twice daily; C, cobimetinib; D, dabrafenib; qd, every day; T, trametinib; V, vemurafenib.

\section{Overall response rate}

For the overall response, testing for interstudy heterogeneity gave significant results $\left(\mathrm{Chi}^{2}=3.45, d f=3[P=0.33], I^{2}=13 \%\right)$; therefore, RR and $95 \% \mathrm{CI}$ were calculated by a fixed-effect model. Our fixed-effect model analysis revealed that combination therapy significantly improved the ORR in comparison to monotherapy (RR: 1.34 [95\% CI: 1.24-1.45], $P<0.00001$ ) as shown in Figure 2A.

\section{Progression-free survival}

For the progression-free survival, testing for interstudy heterogeneity gave significant results $\left(P=0.28, I^{2}=20 \%\right)$, so we used a fixed-effect model for meta-analysis. Our fixed-effects model analysis indicated that PFS in combination therapy was significantly longer than that in monotherapy (HR: 0.58 [95\% CI: 0.52-0.64], $P<0.00001$; Figure 2B).

\section{Overall survival}

Information about HRs for OS was available from six trials, ${ }^{9}, 12-14,16$ which reported that combination therapy showed an improved OS over monotherapy. The pooled HR obtained in our study demonstrated that combination therapy was associated with a significant enhancement of OS compared to monotherapy (HR: 0.70 [95\% CI: $0.62-0.80]$, $P<0.00001$; heterogeneity: $\mathrm{Chi}^{2}=0.26, d f=5[P<0.00001]$, $I^{2}=0 \%$ ) (Figure 2C).

\section{Safety}

$R R$ of all-grade adverse events

The pooled RRs of developing all-grade adverse events (pyrexia, nausea, diarrhea, vomiting, and arthralgia) with combination therapy versus controls were 2.00 (95\% CI: 1.40-2.84), 1.41 (95\% CI: 1.03-1.94), 1.50 (95\% CI: $1.08-2.06), 1.87$ (95\% CI: 1.52-2.31), and $0.71(95 \%$ CI: 0.50-1.02), respectively (Table 2). The results showed a statistically significant increased risk of developing pyrexia, nausea, vomiting, and arthralgia (all $P<0.05$ ) associated with combination therapy compared with monotherapy.

Of the six RCTs ${ }^{9,11-14,16}$ included in the RR analyses, four trials ${ }^{9,11,13,16}$ examined the combination of dabrafenib and trametinib versus dabrafenib alone, one trial ${ }^{12}$ the combination of vemurafenib and cobimetinib versus vemurafenib alone, and one trial the combination of dabrafenib and trametinib versus vemurafenib alone. Subgroup analysis of the RR of all-grade adverse events for combined $B R A F$ and MEK inhibition versus $B R A F$ inhibition alone showed different RRs for pyrexia: combination of dabrafenib and trametinib versus dabrafenib alone $(Z=6.70 ; P<0.00001, \mathrm{RR}=2.22,95 \% \mathrm{CI}$ : 1.76-2.81); combination of vemurafenib and cobimetinib versus vemurafenib alone $(Z=0.98 ; P=0.32, \mathrm{RR}=1.17$, 95\% CI: 0.85-1.61); combination of dabrafenib and trametinib versus vemurafenib alone $(Z=7.96 ; P<0.00001$, $\mathrm{RR}=2.51,95 \%$ CI: 2.00-3.15) (Figure 3A). 
A

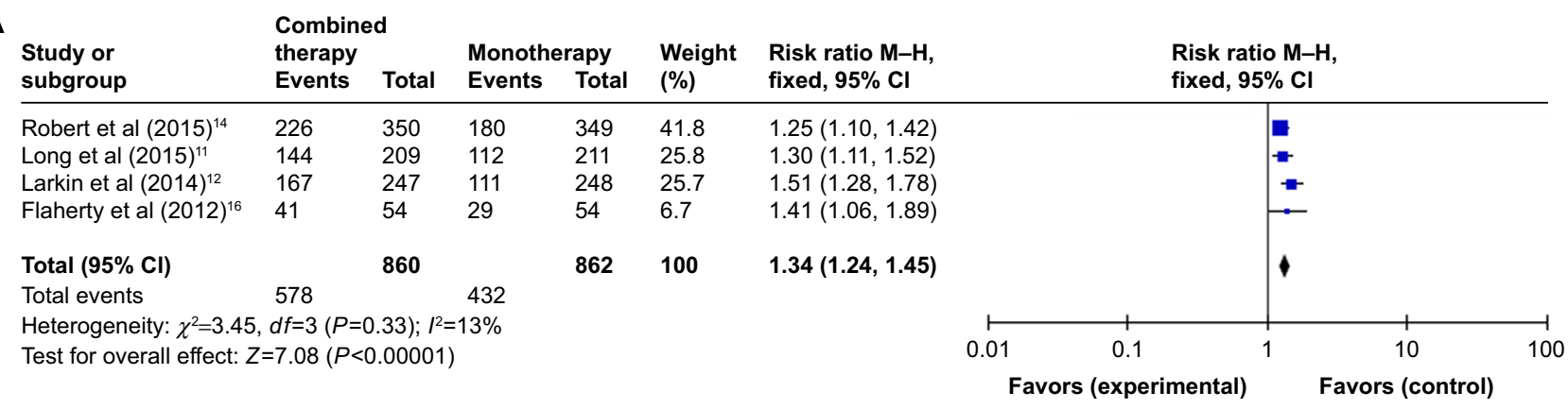

B

Study or
subgroup subgroup Log (hazard ratio)

Robert et al (2015) ${ }^{14} \quad-0.5738$

Schadendorf et al $(2015)^{13}-0.6213$

Long et al (2015) $11 \quad-0.4046$

Long et al (2017) $\quad-0.4321$

Larkin et al (2014) ${ }^{12} \quad-0.6636$

Flaherty et al $(2012)^{16} \quad-0.9322$

$\begin{array}{ll}\text { SE } & \\ 0.1034 & 25 \\ 0.1268 & 17 \\ 0.1175 & 19 . \\ 0.1198 & 19 . \\ 0.1418 & 13 . \\ 0.2317 & 5.1 \\ & \end{array}$

Weight Hazard ratio IV, Hazard ratio IV

Total $(95 \% \mathrm{Cl})$

Heterogeneity: $\chi^{2}=6.23, d f=5(P=0.28) ; l^{2}=20 \%$

Test for overall effect: $Z=10.56(P<0.00001)$

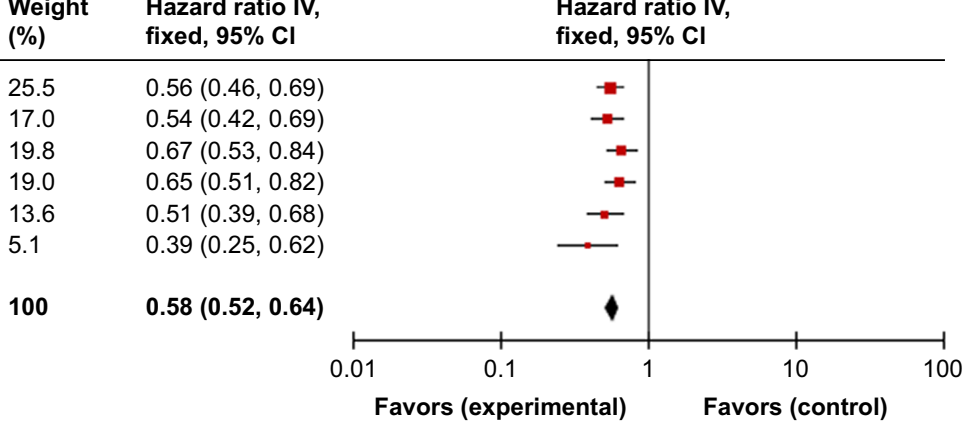

\section{Study or}

subgroup

Log (hazard ratio)

\begin{tabular}{ll} 
SE & $\begin{array}{l}\text { Weight } \\
(\%)\end{array}$ \\
\hline
\end{tabular}

Hazard ratio IV,

fixed, $95 \% \mathrm{Cl}$

Hazard ratio IV,

\begin{tabular}{llll}
\hline Robert et al $(2015)^{14}$ & -0.4421 & 0.3925 & 3.0
\end{tabular}

Schadendorf et al $(2015)^{13}-0.3254$

Long et al (2015) $\quad-0.3416$

Long et al $(2017)^{9} \quad-0.3512$

Larkin et al (2014) $\quad-0.4338$

Flaherty et al $(2012)^{16} \quad-0.3934$

$0.1168 \quad 34.1$

$0.1312 \quad 27.0$

$0.1437 \quad 22.5$

$0.2213 \quad 9.5$

$0.3497 \quad 3.8$

$0.64(0.30,1.39)$

$0.72(0.57,0.91)$

$0.71(0.55,0.92)$

$0.70(0.53,0.93)$

$0.65(0.42,1.00)$

$0.67(0.34,1.34)$

Total $(95 \% \mathrm{Cl})$

Heterogeneity: $\chi^{2}=0.26, d f=5(P=1.00) ; l^{2}=0 \%$

Test for overall effect: $Z=5.16(P<0.00001)$

100

$0.70(0.62,0.80)$ fixed, $95 \% \mathrm{Cl}$

Figure 2 Forest plot analysis of the efficiency outcomes of combined BRAF and MEK inhibition versus BRAF inhibition alone. (A) Overall response rate, (B) progression-free survival, and (C) overall survival.

Abbreviations: $\mathrm{Cl}$, confidence interval; IV, inverse variance; $\mathrm{M}-\mathrm{H}$, Mantel-Haenszel; SE, standard error.

Regarding the RR of nausea events, combination of dabrafenib and trametinib versus dabrafenib $(Z=2.06$; $P=0.04, \mathrm{RR}=1.64,95 \% \mathrm{CI}: 1.03-2.62)$ and combination of vemurafenib and cobimetinib versus vemurafenib $(Z=3.52$; $P=0.004, \mathrm{RR}=1.63,95 \% \mathrm{CI}: 1.24-2.15)$ showed a significant difference, while combination of dabrafenib and trametinib versus vemurafenib showed no significant difference $(Z=0.29$; $P=0.77, \mathrm{RR}=1.03,95 \%$ CI: $0.84-1.26$ ) (Figure 3B). Regarding the RR of diarrhea, combination of vemurafenib and cobimetinib versus vemurafenib alone and combination of dabrafenib and trametinib versus vemurafenib alone showed significant results $(Z=6.01 ; P<0.00001, \mathrm{RR}=2.02,95 \% \mathrm{CI}$ : 1.61-2.54; $Z=2.01 ; P=0.04, \mathrm{RR}=1.23,95 \%$ CI: $1.01-1.50$ ), whereas combination of dabrafenib and trametinib versus dabrafenib alone showed no significant results $(Z=1.13$; $P=0.26, \mathrm{RR}=1.44,95 \% \mathrm{CI}: 0.76-2.71$ ) (Figure 3C).
Regarding vomiting, significant differences were found between combination of dabrafenib and trametinib versus dabrafenib, combination of vemurafenib and cobimetinib versus vemurafenib, and combination of dabrafenib with trametinib versus vemurafenib $(Z=3.13 ; P=0.002, \mathrm{RR}=1.94$, 95\% CI: $1.28-2.94 ; Z=2.65 ; P=0.008, \mathrm{RR}=1.75,95 \% \mathrm{CI}$ : $1.16-2.25 ; Z=4.23 ; P<0.0001, \mathrm{RR}=1.90,95 \%$ CI: $1.41-2.56$, respectively) (Figure 3D).

Regarding RR of arthralgia, combination of dabrafenib and trametinib versus vemurafenib showed a significant difference $(Z=6.94 ; P<0.0001, \mathrm{RR}=0.47,95 \%$ CI: $0.55-1.00)$. No significant differences were observed between combination of dabrafenib and trametinib versus dabrafenib and combination of vemurafenib and cobimetinib versus vemurafenib $(Z=0.82 ; P=0.41, \mathrm{RR}=0.85,95 \% \mathrm{CI}: 0.57-1.26 ; Z=1.72$; $P=0.08, \mathrm{RR}=0.81,95 \%$ CI: 0.64-1.03) (Figure 3E). 
A

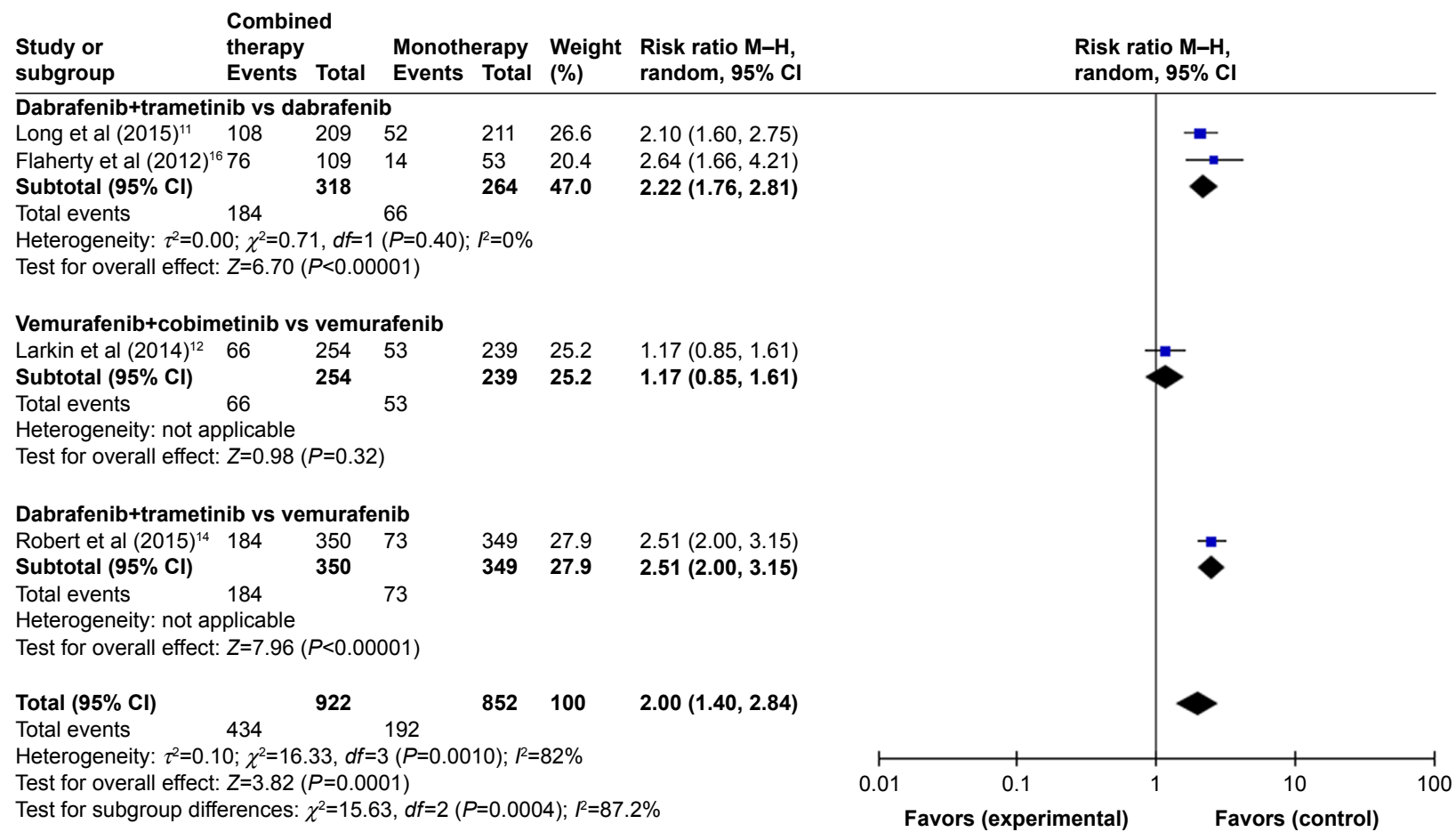

B

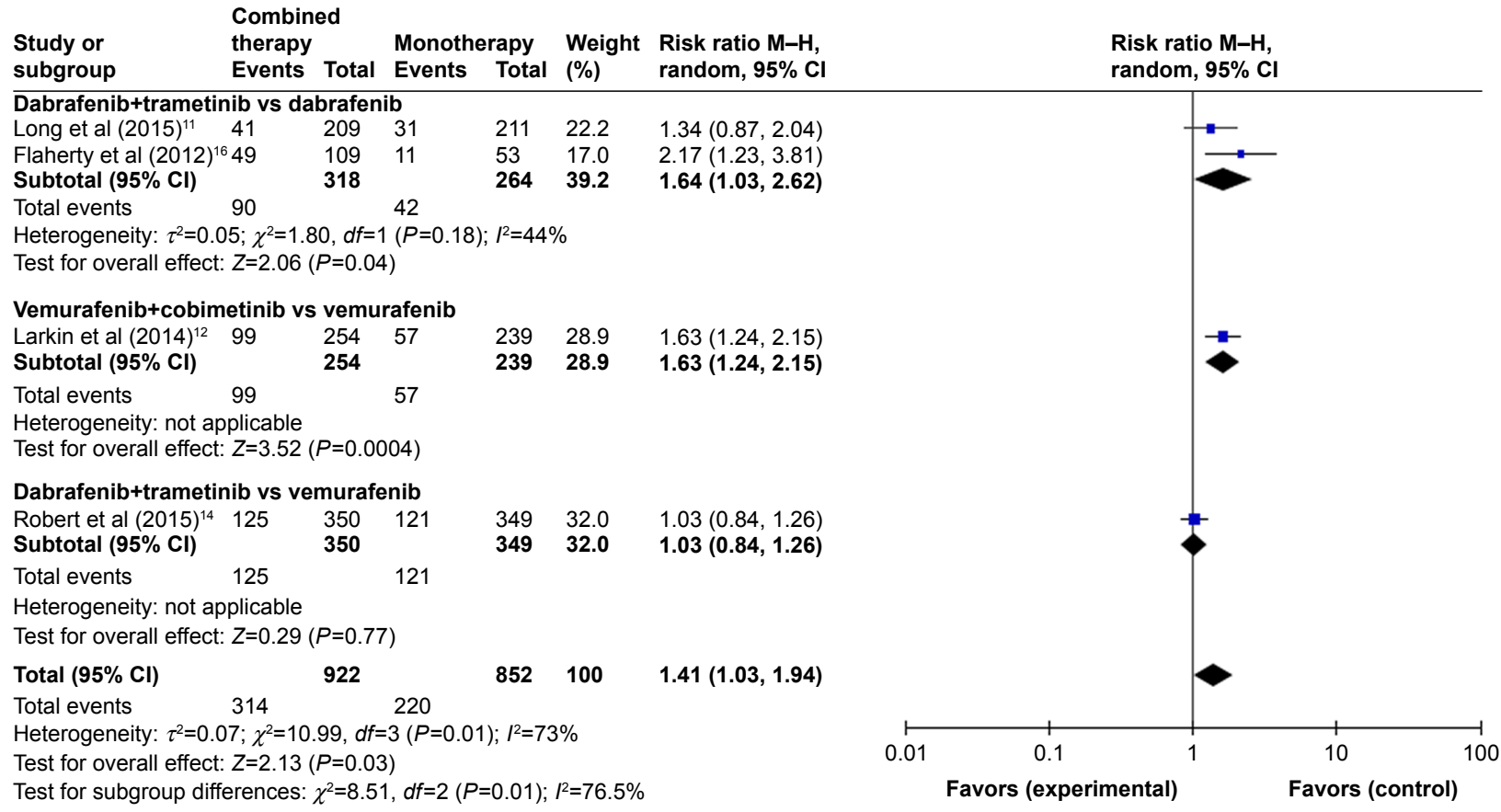

Figure 3 (Continued) 


\section{C}

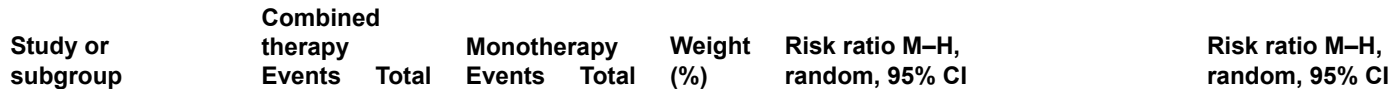

\begin{tabular}{llllllll}
\hline Dabrafenib+trametinib vs dabrafenib & & & & \\
Long et al $(2015)^{11}$ & 38 & 209 & 19 & 211 & 17.6 & $2.02(1.20,3.38)$ \\
Flaherty et al $(2012)^{16}$ & 68 & 109 & 30 & 53 & 26.0 & $1.10(0.84,1.45)$ \\
\hline
\end{tabular}

$\begin{array}{lllll}\text { Subtotal }(95 \% \mathrm{Cl}) & 318 & 264 & 43.6 & 1.44(0.76,2.71)\end{array}$

Total events $\quad 106 \quad 49$

Heterogeneity: $\tau^{2}=0.17 ; \chi^{2}=4.74, d f=1(P=0.03) ; I^{2}=79 \%$

Test for overall effect: $Z=1.13(P=0.26)$

Vemurafenib+cobimetinib vs vemurafenib

$\begin{array}{lllllll}\text { Larkin et al }(2014)^{12} & 144 & 254 & 67 & 239 & 27.7 & 2.02(1.61,2.54) \\ \text { Subtotal }(\mathbf{9 5 \%} \mathbf{C l}) & & \mathbf{2 5 4} & & \mathbf{2 3 9} & \mathbf{2 7 . 7} & \mathbf{2 . 0 2}(\mathbf{1 . 6 1 , 2 . 5 4 )}\end{array}$

Total events $\quad 144 \quad 67$

Heterogeneity: not applicable

Test for overall effect: $Z=6.01(P<0.00001)$

Dabrafenib+trametinib vs vemurafenib

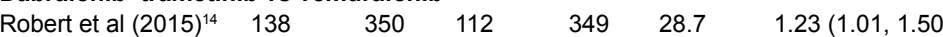

$\begin{array}{llllll}\text { Subtotal }(\mathbf{9 5 \%} \mathrm{Cl}) & \mathbf{3 5 0} & & 349 & 28.7 & 1.23(1.01,1.50) \\ & & \mathbf{3 4 9} & \mathbf{2 8 . 7} & \mathbf{1 . 2 3}(\mathbf{1 . 0 1}, \mathbf{1 . 5 0})\end{array}$

Total events $\quad 138$

Heterogeneity: not applicable

Test for overall effect: $Z=2.01(P=0.04)$

Total $(95 \% \mathrm{Cl})$

Total events $\quad 388 \quad 228$

Heterogeneity: $\tau^{2}=0.08 ; \chi^{2}=16.30, d f=3(P=0.0010) ; I^{2}=82 \%$

Test for overall effect: $Z=2.46(P=0.01)$

Test for subgroup differences: $\chi^{2}=10.29, d f=2(P=0.006) ; I^{2}=80.6 \%$

$1.50(1.08,2.06)$

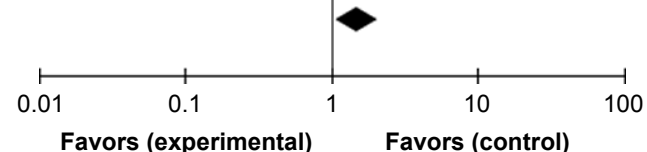

D

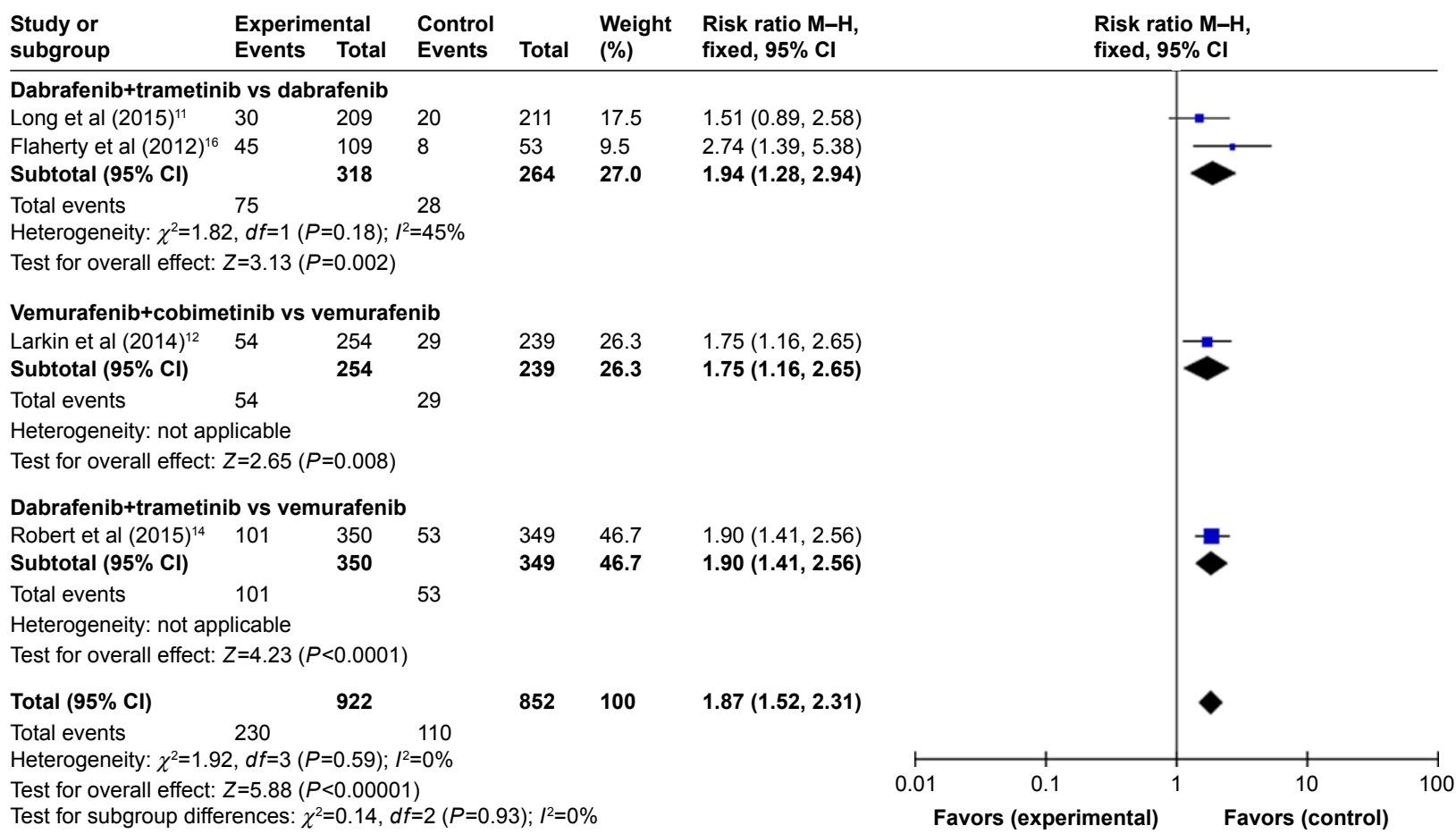

Figure 3 (Continued) 


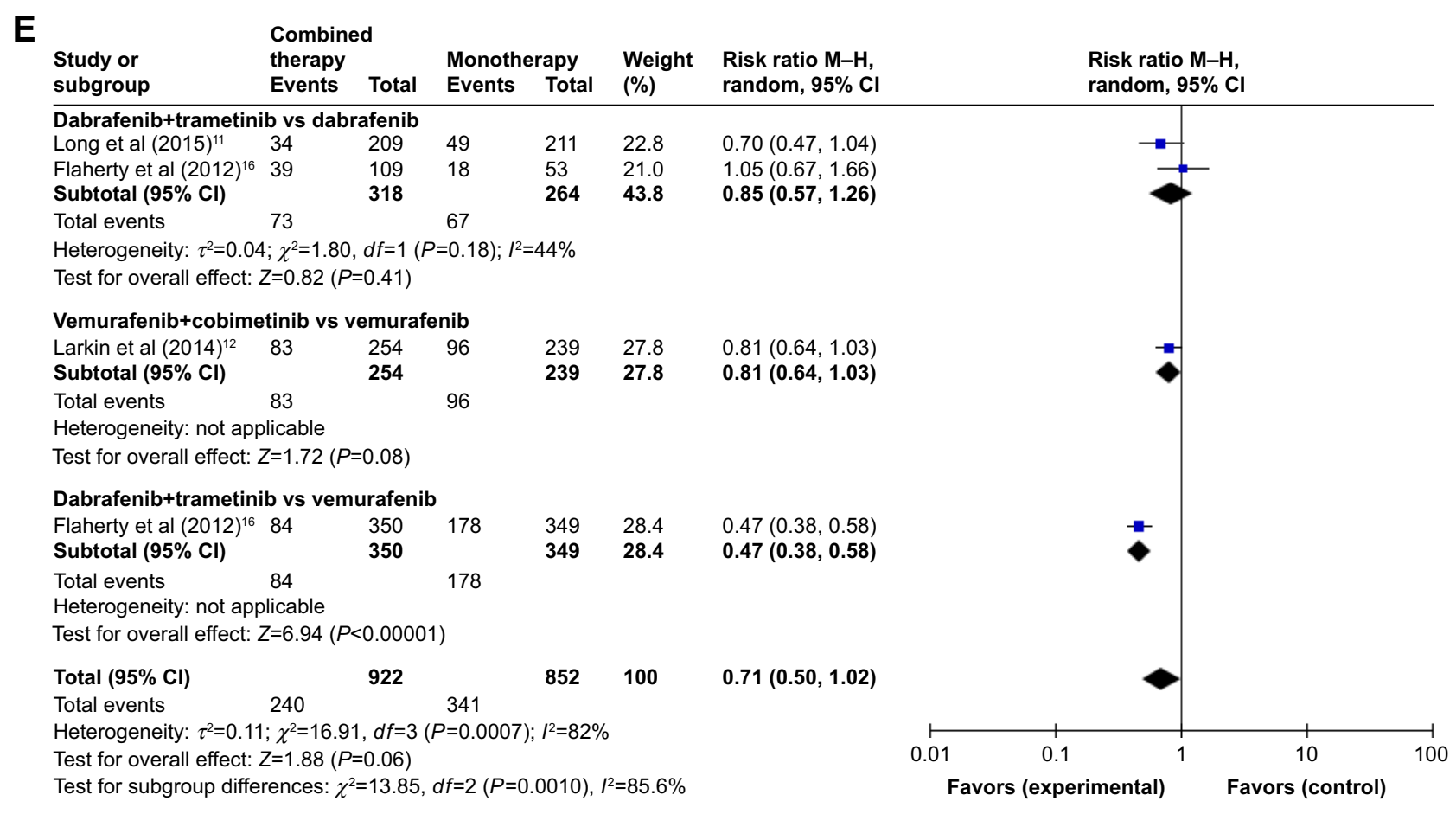

Figure 3 Subgroup analysis of the relative risk (RR) of all-grade adverse events for combined BRAF and MEK inhibition versus BRAF inhibition alone. (A) Pyrexia, (B) nausea, (C) diarrhea, (D) vomiting, and (E) arthralgia.

Abbreviations: $\mathrm{Cl}$, confidence interval; IV, inverse variance; $\mathrm{M}-\mathrm{H}$, Mantel-Haenszel; SE, standard error.

Table 2 RR of adverse events between combined targeted therapy and monotherapy

\begin{tabular}{|c|c|c|c|c|c|c|}
\hline \multirow[t]{2}{*}{ Subgroup } & \multicolumn{2}{|l|}{ Control } & \multirow{2}{*}{$\begin{array}{l}\text { Analysis } \\
\text { number }\end{array}$} & \multirow{2}{*}{$\begin{array}{l}\text { All-grade } \\
\text { RR }\end{array}$} & \multirow[t]{2}{*}{$95 \% \mathrm{Cl}$} & \multirow[t]{2}{*}{$P$-value } \\
\hline & $\begin{array}{l}\text { Combined } \\
\text { therapy }\end{array}$ & Monotherapy & & & & \\
\hline \multicolumn{7}{|l|}{ Pyrexia } \\
\hline Combined BRAF and MEK inhibition vs BRAF alone & 922 & 852 & $11-14,16$ & 2.00 & $1.40-2.84$ & 0.0001 \\
\hline Dabrafenib+trametinib vs dabrafenib & 318 & 264 & 11,16 & 2.22 & I.76-2.8I & $<0.00001$ \\
\hline Vemurafenib+cobimetinib vs vemurafenib & 254 & 239 & 12 & 1.17 & $0.85-1.61$ & 0.32 \\
\hline Dabrafenib+trametinib vs vemurafenib & 350 & 349 & 14 & 2.51 & $2.00-3.15$ & $<0.00001$ \\
\hline \multicolumn{7}{|l|}{ Nausea } \\
\hline Combined BRAF and MEK inhibition vs BRAF alone & 922 & 852 & $11-14,16$ & 1.41 & $1.03-1.94$ & 0.03 \\
\hline Dabrafenib+trametinib vs dabrafenib & 318 & 264 & 11,16 & 1.64 & $1.03-2.62$ & 0.04 \\
\hline Vemurafenib+cobimetinib vs vemurafenib & 254 & 239 & 12 & 1.63 & $1.24-2.15$ & 0.0004 \\
\hline Dabrafenib+trametinib vs vemurafenib & 350 & 349 & 14 & 1.03 & $0.84-1.26$ & 0.77 \\
\hline \multicolumn{7}{|l|}{ Diarrhea } \\
\hline Combined BRAF and MEK inhibition vs BRAF alone & 922 & 852 & $11-14,16$ & 1.50 & $1.08-2.06$ & 0.01 \\
\hline Dabrafenib+trametinib vs dabrafenib & 527 & 475 & 11,16 & 1.44 & $0.76-2.71$ & 0.26 \\
\hline Vemurafenib+cobimetinib vs vemurafenib & 254 & 239 & 12 & 2.02 & $\mathrm{I} .6 \mathrm{I}-2.54$ & $<0.0001$ \\
\hline Dabrafenib+trametinib vs vemurafenib & 350 & 349 & 14 & 1.23 & $1.01-1.50$ & 0.04 \\
\hline \multicolumn{7}{|l|}{ Vomiting } \\
\hline Combined BRAF and MEK inhibition vs BRAF alone & 922 & 852 & $11-14,16$ & 1.87 & $|.52-2.3|$ & $<0.0001$ \\
\hline Dabrafenib+trametinib vs dabrafenib & 318 & 264 & 11,16 & 1.94 & $1.28-2.94$ & 0.002 \\
\hline Vemurafenib+cobimetinib vs vemurafenib & 254 & 239 & 12 & 1.75 & $1.16-2.65$ & 0.008 \\
\hline Dabrafenib+trametinib vs vemurafenib & 350 & 349 & 14 & 1.90 & $\mathrm{I} .4 \mathrm{I}-2.56$ & $<0.0001$ \\
\hline \multicolumn{7}{|l|}{ Arthralgia } \\
\hline Combined BRAF and MEK inhibition vs BRAF alone & 922 & 852 & $11-14,16$ & 0.71 & $0.50-1.02$ & 0.06 \\
\hline Dabrafenib+trametinib vs dabrafenib & 527 & 475 & 11,16 & 0.85 & $0.57-1.26$ & 0.17 \\
\hline Vemurafenib+cobimetinib vs vemurafenib & 254 & 239 & 12 & 0.81 & $0.64-1.03$ & 0.08 \\
\hline Dabrafenib+trametinib vs vemurafenib & 350 & 349 & 14 & 0.47 & $0.38-0.58$ & $<0.0001$ \\
\hline
\end{tabular}

Abbreviations: RR, relative risk; MEK, mitogen-activated extracellular signal-regulated kinase. 


\section{Incidence of all-grade adverse events associated with combined BRAF and MEK inhibition}

Results of the random-effect model showed that the pooled incidence of all-grade adverse events associated with combined $B R A F$ and MEK inhibition of all the included trials was 50\% (95\% CI: $37 \%-62 \%$ ), $38 \%$ (95\% CI: $29 \%-49 \%$ ), $39 \%$ (95\% CI: 26\%-54\%), 29\% (95\% CI: $21 \%-39 \%$ ), and 24\% (95\% CI: 18\%-32\%), respectively (Table 3).

As meta-analysis results about adverse events revealed heterogeneity, we further performed subgroup analysis according to the treatment regimens. All the studies were classified into two subgroups: 1) combined dabrafenib and trametinib and 2) combined vemurafenib and cobimetinib. By subgroup analysis of the incidences of combined $B R A F$ and MEK inhibition-associated all-grade adverse events stratified by treatment regimen, we found higher overall incidences of all-grade pyrexia were observed in patients on combined dabrafenib and trametinib (55\%, 95\% CI: $46 \%-64 \%)$ and patients on combined vemurafenib and cobimetinib $(27 \%$, 95\% CI: 22\%-33\%) (Figure 4A).

Different overall incidences of all-grade nausea were observed in patients treated with combination of dabrafenib and trametinib (39\%, 95\% CI: $27 \%-52 \%)$ and combination of vemurafenib and cobimetinib (39\%, 95\% CI: 33\%-45\%) (Figure 4B). Furthermore, higher incidences of all-grade diarrhea were observed in patients treated with combination of vemurafenib and cobimetinib (57\%, 95\% CI: 51\%-63\%) followed by those treated with combination of dabrafenib and trametinib (36\%, 95\% CI: 22\%-53\%) (Figure 4C).

The incidence of all-grade vomiting events was more higher in patients treated with combination of dabrafenib and trametinib (31\%, 95\% CI: $21 \%-41 \%)$ followed by combination of vemurafenib and cobimetinib (21\%, 95\% CI: 17\%-27\%) (Figure 4D). Finally, the overall incidences of all-grade arthralgia were more higher in patients treated with combination of vemurafenib and cobimetinib (30\%, 95\% CI: $24 \%-35 \%)$ followed by combination of dabrafenib and trametinib (22\%, 95\% CI: $14 \%-32 \%)$ (Figure 4E).

\section{Publication bias}

Publication bias was assessed using funnel plot produced by Review Manager 5.3 software. There was an obvious asymmetry of the funnel plot (Figure 5), which suggests that there was some level of publication bias. However, because the number of included studies was only eight, the funnel plots may not be significant. Egger's test revealed that publication bias was not significant for both the incidence and RR of adverse events (incidence: $P=0.29$; RR: $P=0.51$ ).

\section{Discussion}

For metastatic melanoma patients with activating $B R A F$ mutations, the treatment standard is $B R A F$-targeted therapy,

Table 3 Overall incidence of all-grade adverse events in patients receiving combined targeted therapy

\begin{tabular}{|c|c|c|c|c|c|}
\hline \multirow[t]{2}{*}{ Subgroup } & \multicolumn{2}{|c|}{ Sample size } & \multirow{2}{*}{$\begin{array}{l}\text { Analysis } \\
\text { number }\end{array}$} & \multirow{2}{*}{$\begin{array}{l}\text { All-grade } \\
\text { incidence }\end{array}$} & \multirow[t]{2}{*}{$95 \% \mathrm{Cl}$} \\
\hline & Events & Total & & & \\
\hline \multicolumn{6}{|l|}{ Pyrexia } \\
\hline Combined BRAF and MEK inhibition & 477 & 1,003 & $10-16$ & 0.50 & $0.37-0.62$ \\
\hline Dabrafenib+trametinib & 411 & 756 & $10-11,14-16$ & 0.55 & $0.46-0.64$ \\
\hline Vemurafenib+cobimetinib & 66 & 247 & 12 & 0.27 & $0.22-0.33$ \\
\hline \multicolumn{6}{|l|}{ Nausea } \\
\hline Combined BRAF and MEK inhibition & 351 & 991 & $10-16$ & 0.38 & $0.29-0.49$ \\
\hline Dabrafenib+trametinib & 252 & 737 & $10-11,14-16$ & 0.39 & $0.27-0.52$ \\
\hline Vemurafenib+cobimetinib & 99 & 254 & 12 & 0.39 & $0.33-0.45$ \\
\hline \multicolumn{6}{|l|}{ Diarrhea } \\
\hline Combined BRAF and MEK inhibition & 410 & 991 & $10-16$ & 0.39 & $0.26-0.54$ \\
\hline Dabrafenib+trametinib & 266 & 737 & $10-11,14-16$ & 0.36 & $0.22-0.53$ \\
\hline Vemurafenib+cobimetinib & 144 & 254 & 12 & 0.57 & $0.5 \mathrm{I}-0.63$ \\
\hline \multicolumn{6}{|l|}{ Vomiting } \\
\hline Combined BRAF and MEK inhibition & 166 & 991 & $10-12,14-16$ & 0.29 & $0.21-0.39$ \\
\hline Dabrafenib+trametinib & 205 & 737 & $10-11,14-16$ & 0.31 & $0.21-0.4 I$ \\
\hline Vemurafenib+cobimetinib & 54 & 254 & 12 & 0.21 & $0.17-0.27$ \\
\hline \multicolumn{6}{|l|}{ Arthralgia } \\
\hline Combined BRAF and MEK inhibition & 213 & $\mathrm{I}, 157$ & $10-12,14,16$ & 0.24 & $0.18-0.32$ \\
\hline Dabrafenib+trametinib & 159 & 694 & $10-11,14-16$ & 0.22 & $0.14-0.32$ \\
\hline Vemurafenib+cobimetinib & 54 & 254 & 12 & 0.30 & $0.24-0.35$ \\
\hline
\end{tabular}


A

\begin{tabular}{l} 
Study \\
\hline Subgroup variables: dabrafenib+tramet \\
Douglas (2014) \\
Long et al $(2015)^{11}$
\end{tabular}

Heterogeneity: $I^{2}=78.2 \%, \tau^{2}=0.1176, P=0.001$

Subgroup variables: vemurafenib+cobimetinib Larkin et al $2014^{12}$

Random effects model

Heterogeneity: $I^{2}=\mathrm{NaN} \%, \tau^{2}=0, P=1$

Random effects model

Heterogeneity: $I^{2}=93 \%, \tau^{2}=0.3834, P<0.0001$ $66 \quad 247$

Events

16

108

184

27

7

\section{inib}

Total

45

209

109

43

756

247
247

1,003

$\begin{array}{llllllllll}0.1 & 0.2 & 0.3 & 0.4 & 0.5 & 0.6 & 0.7 & 0.8\end{array}$

Proportion

$95 \% \mathrm{Cl}$ W (random)

Proportion

$\begin{array}{ll}0.36(0.22,0.51) & 14.9 \% \\ 0.52(0.45,0.59) & 17.8 \%\end{array}$

$0.27(0.21,0.33) \quad 18.1 \%$

$0.53(0.47,0.58) \quad 16.7 \%$

$0.70(0.60,0.78) \quad 14.8 \%$

$0.55(0.46,0.64) \quad 82.3 \%$

$0.63(0.47,0.77) \quad 17.7 \%$

$0.27(0.22,0.33) \quad 17.7 \%$

$0.50(0.37,0.62) \quad 100 \%$

B

\begin{tabular}{lcc} 
Study & Events & Tot \\
\hline Subgroup variables: dabrafenib+trametinib & \\
Douglas $(2014)$ & 10 \\
${\text { Long et al }(2015)^{11}}^{11}$ & 41 & 26 \\
Robert et al $(2015)^{14}$ & 125 & 350 \\
Flaherty et al $(2012)^{16}$ & 49 \\
${\text { Corcoran et al }(2015)^{15}}_{\text {Random effects model }}$ & 27 \\
\hline
\end{tabular}

Heterogeneity: $I^{2}=89.6 \%, \tau^{2}=0.3372, P<0.0001$

Subgroup variables: vemurafenib+cobimetinib Larkin et al $2014^{12}$

99

254

Total

Proportion

$95 \% \mathrm{Cl}$

W (random)

Random effects model

254

Heterogeneity: $I^{2}=\mathrm{NaN} \%, \tau^{2}=0, P=1$

Random effects model

Heterogeneity: $I^{2}=87.5 \%, \tau^{2}=0.2164, P<0.0001$

991

209

109

43

737

90

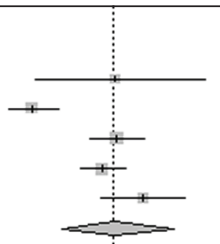

$0.38(0.20,0.59)$

$\quad 18.0 \%$

$0.39(0.33,0.45) \quad 19.5 \%$

$0.36(0.31,0.41) \quad 17.6 \%$

$0.45(0.35,0.55) \quad 14.1 \%$

$0.39(0.27,0.52) \quad 80.9 \%$

$0.63(0.47,0.77) \quad 19.1 \%$

$0.39(0.33,0.45) \quad 19.1 \%$

$0.38(0.29,0.49) \quad 100 \%$

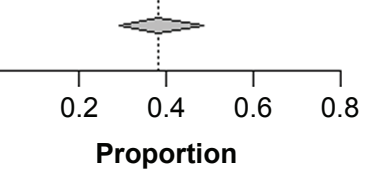

C

\begin{tabular}{lc} 
Study & Event \\
\hline Subgroup variables: dabrafenib+trametinib \\
Douglas (2014) & 7 \\
Long et al $(2015)^{11}$ & 38 \\
Robert et al $(2015)^{14}$ & 138 \\
Flaherty et al $(2012)^{16}$ & 68 \\
Corcoran et al $(2015)^{15}$ & 15 \\
Random effects model &
\end{tabular}

Random effects model

Heterogeneity: $I^{2}=93.2 \%, \tau^{2}=0.5562, P=0.0001$

Subgroup variables: vemurafenib+cobimetinib

Larkin et al $2014^{12}$

$144 \quad 254$

Random effects model

Heterogeneity: $I^{2}=\mathrm{NaN} \%, \tau^{2}=0, P=1$

Random effects model

Heterogeneity: $I^{2}=94.2 \%, \tau^{2}=0.513, P<0.0001$

Total

Proportion

$95 \% \mathrm{Cl}$

W (random)

26
209
350
109
43
737

737

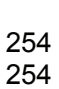

254

991

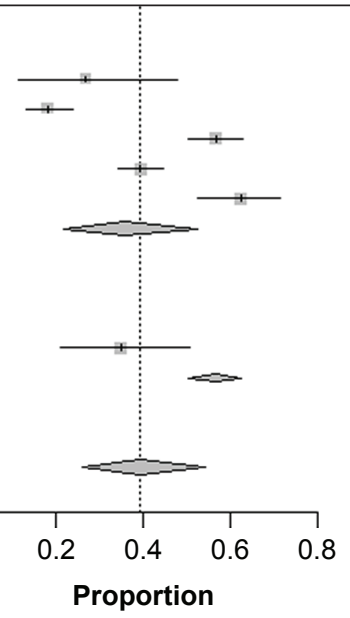

$0.27(0.12,0.48)$

$17.5 \%$

$0.57(0.50,0.63) \quad 18.2 \%$

$0.39(0.34,0.45) \quad 17.3 \%$

$0.62(0.53,0.71) \quad 15.5 \%$

$0.36(0.22,0.53) \quad 82.0 \%$

$0.35(0.21,0.51) \quad 18.0 \%$

$0.57(0.51,0.63) \quad 18.0 \%$

$0.39(0.26,0.54) \quad 100 \%$

Figure 4 (Continued) 
D

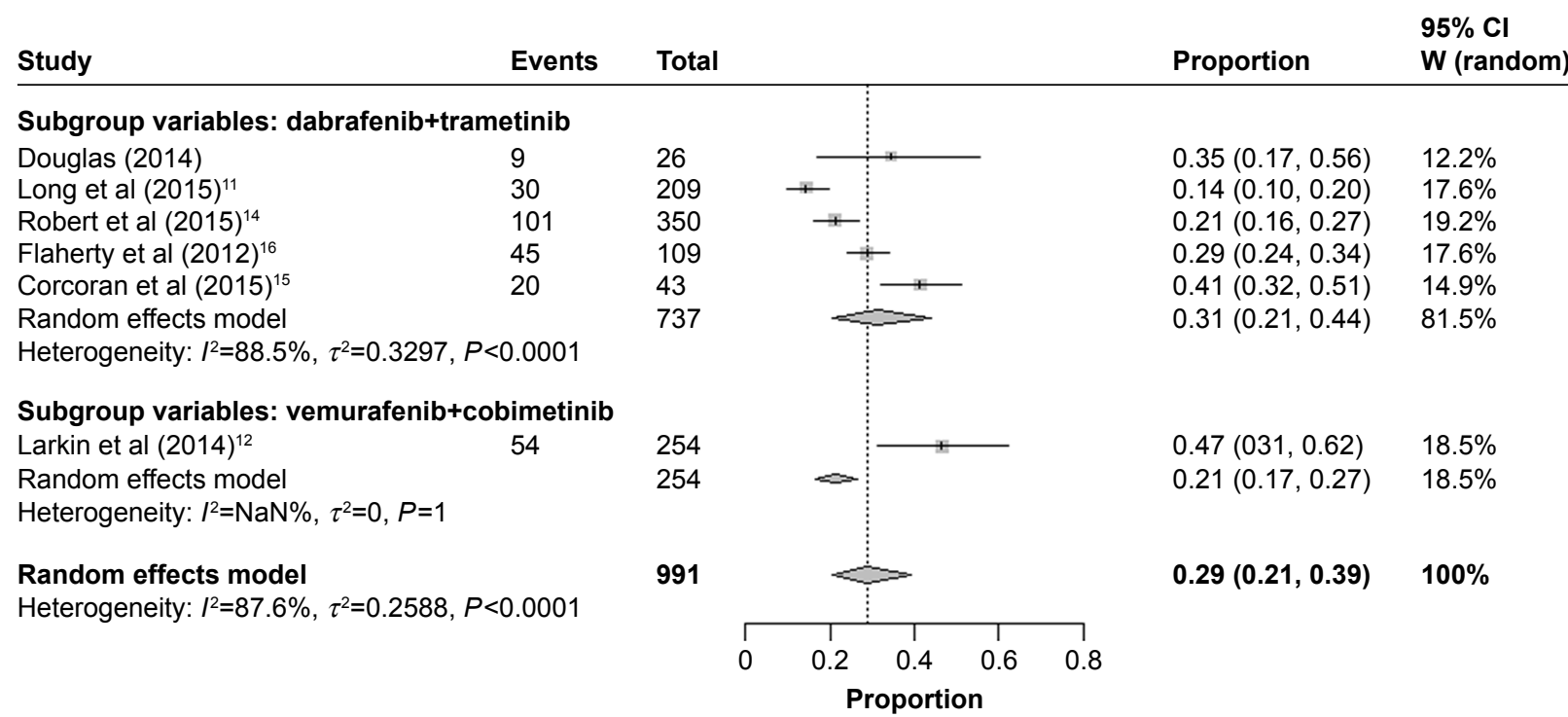

E

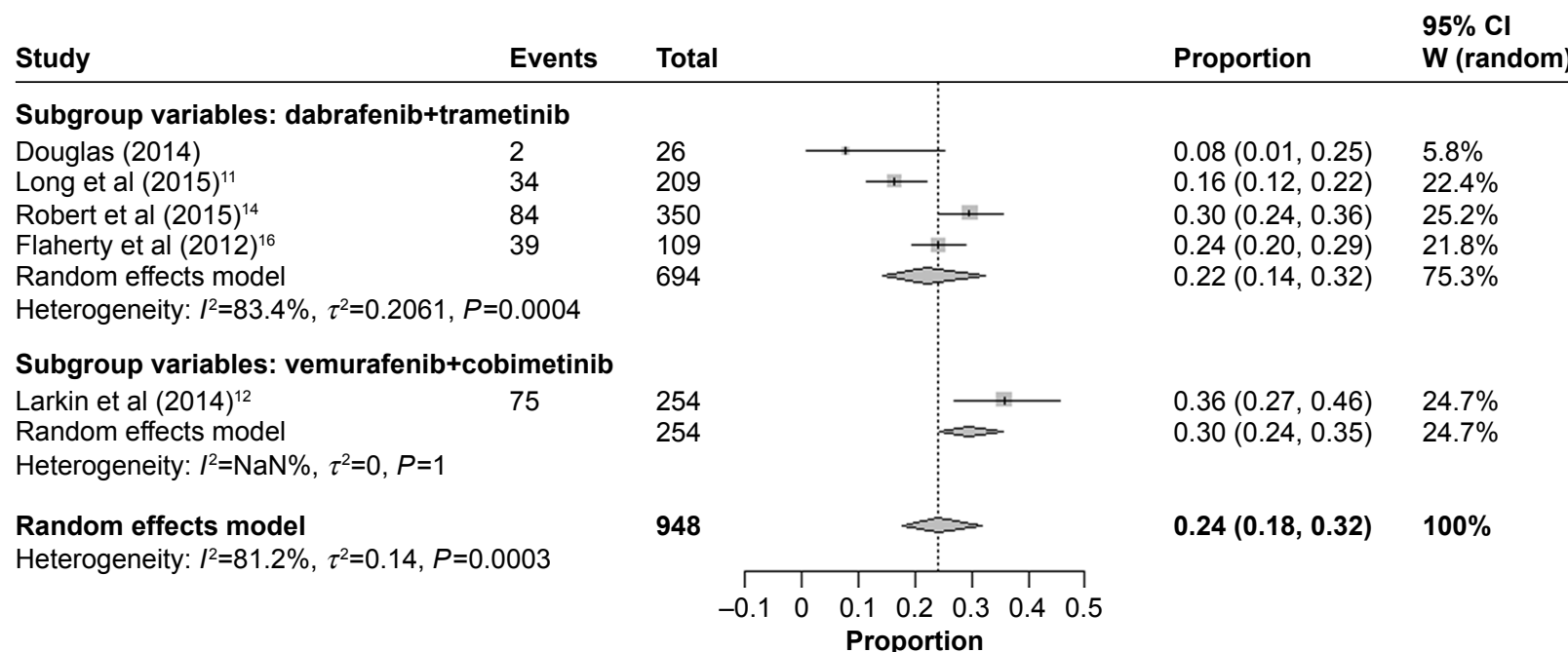

Figure 4 Subgroup analysis of the incidence of all-grade adverse events for combined BRAF and MEK inhibition. (A) Pyrexia, (B) nausea, (C) diarrhea, (D) vomiting, and (E) arthralgia.

Abbreviations: $\mathrm{Cl}$, confidence interval; $\mathrm{W}$, weight.

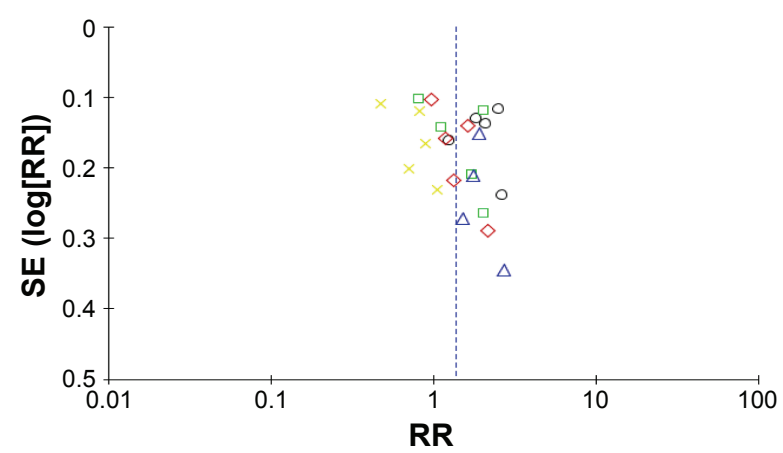

Subgroups

$\bigcirc$ Pyrexia $\diamond$ Nausea $\square$ Diarrhea $\triangle$ Vomiting $\times$ Arthralgia

Figure 5 Funnel plot analysis for publication bias assessment.

Abbreviations: RR, relative risk; SE, standard error. which is based on improvement in the rate of survival, compared with conventional chemotherapy. ${ }^{17}$ However, clinical evidence shows that resistance occurs in 6-7 months after the initiation of treatment. Several mechanisms of MAPK-dependent resistance to $B R A F$ inhibitors have been described in vitro and corroborated in tumor specimens obtained from patients. It was hypothesized that inhibition of the MAPK pathway downstream of $B R A F$ could suppress mechanisms of resistance. ${ }^{18}$ In recent years, lots of clinical data suggest that combined $B R A F$ and MEK inhibition can block paradoxical activation of the MAPK pathway induced by single-agent $B R A F$ inhibitors, and an opportunity to investigate a regimen combining a $B R A F$ inhibitor with an 
MEK inhibitor was provided by the fact of MEK inhibition having been validated as a therapeutic approach in the same patient population. ${ }^{19}$ However, it has been found that sequential use of an MEK inhibitor after progression on a $B R A F$ inhibitor does not result in clinically meaningful antitumor activity either in vitro or in patients. Concurrent administration of MEK and $B R A F$ inhibitors is effective on cell lines with acquired resistance to $B R A F$ inhibitors and can achieve responses in patients with $B R A F$ V600-mutant melanoma progressing on single-agent BRAF inhibitors. ${ }^{20}$ More importantly, many studies have confirmed that for patients who have not previously received a $B R A F$ inhibitor, treatment with a combination of a BRAF inhibitor and an MEK inhibitor could lead to greater initial antitumor activity and could prevent or delay MAPK-driven acquired resistance mechanisms. ${ }^{21}$ Consistent with this, the results of our metaanalysis also demonstrated that combined $B R A F$ and MEK inhibition provided significant advantage in ORR, PFS, and OS over MEK inhibition alone. However, the reported success of these agents comes at the cost of a set of adverse events, which significantly can affect therapeutic effect and quality of life of patients, and can lead to infection, discomfort, and bring some mental burden for patients to a certain degree. ${ }^{22}$ Thus, a comprehensive analysis of all these reported adverse events has been conducted in our study.

The results of our meta-analysis showed that the group treated with combination of $B R A F$ and MEK inhibitors exhibited superior results compared to that treated with $B R A F$ alone: ORR - combined RR $=1.34,95 \% \mathrm{CI}: 1.24-1.45$, $P<0.00001$; PFS - combined HR $=0.58,95 \%$ CI: $0.52-0.64$, $P<0.00001$ ); and OS rate - combined $\mathrm{HR}=0.70,95 \% \mathrm{CI}$ : $0.62-0.80, P<0.00001$. Despite these advantages, combined therapy with $B R A F$ and MEK inhibitors revealed some additional toxicities. To our knowledge, this is the first meta-analysis focusing specifically on the adverse events associated with the combination therapy. Our meta-analysis was able to demonstrate that combination therapy was associated with a significant increase in the risk of adverse events such as pyrexia, nausea, diarrhea, and vomiting in patients with melanoma. The most frequent treatment-related allgrade adverse event was pyrexia, while diarrhea was the less common. ${ }^{23}$ Based on our further subgroup analysis, it was not difficult to find that combined vemurafenib and cobimetinib therapy may pose a higher risk of developing nausea and diarrhea compared to the combined dabrafenib and trametinib therapy. ${ }^{24}$ Moreover, subgroup analysis also showed that combined vemurafenib and cobimetinib therapy may carry lower risks of developing pyrexia and vomiting than the combined dabrafenib and trametinib therapy.
Based on our results, we also found that patients receiving combination therapy had more frequent gastrointestinal toxic effects (eg, nausea, vomiting, and diarrhea) in comparison with patients receiving $B R A F$ inhibitor alone. ${ }^{25} \mathrm{We}$ believe that it is very import to use nonpharmacological and pharmacological management to adjust treatment and therapeutic regimens according to the severity and types of gastrointestinal events, because pain and discomfort of patients can be alleviated by adequate prevention and treatment. ${ }^{26}$ Firstly, it is advised that patients should realize the importance of managing these adverse events at the early treatment phase and strictly avoiding combined $B R A F$ and MEK inhibition discontinuation. Secondly, the health care team of cancer patients should be informed to be ready for nutritional advice so to avoid hyponatremia or hypokalemia when gastrointestinal events occur. ${ }^{27}$ Thirdly, gastrointestinal protective agents such as loperamide, omeprazole, and ondansetron should be well prepared before patients receive combination therapy; these agents should be immediately started at the onset of gastrointestinal symptoms. ${ }^{28}$

Our meta-analysis had several limitations. Firstly, only eight studies met our inclusion criteria, and hence the small number of trials and low quality of most of the work could make the conclusion less convincing; also publication bias could not be completely excluded based on funnel plot. ${ }^{29}$ Secondly, because most researchers applied their personal experience in diagnosing the toxicities in the clinical trials, there were different judgements based on the same signs. Thirdly, the treatment regimens and doses of drugs are different among the studies included in the meta-analysis, which led to significant heterogeneity of the data. Therefore, largescale and well-designed studies are needed to summarize and analyze the data to draw a more convincing conclusion. ${ }^{30}$

\section{Conclusion}

In summary, this study shows that combined therapy of $B R A F$ and MEK inhibitors may moderately improve the overall response, PFS, and OS, although it may increase the incidence of some adverse events. In addition, prompt and effective management of these adverse events might allow for the safer use of combination therapy. We believe that our results could provide a reference point for physicians in clinical practice and ensure the safety and efficacy of treatment regimen for melanoma patients.

\section{Disclosure}

The authors report no conflicts of interest in this work. 


\section{References}

1. Martinliberal J, Larkin J. Vemurafenib for the treatment of BRAF mutant metastatic melanoma. Future Oncol. 2015;11(4):579-589.

2. Shi H, Hugo W, Kong X, et al. Acquired resistance and clonal evolution in melanoma during BRAF inhibitor therapy. Cancer Discov. 2014; 4(1):80-93.

3. Chapman PB, Hauschild A, Robert C, et al. Improved survival with vemurafenib in melanoma with BRAF V600E mutation. N Engl J Med. 2011;364(26):2507-2516.

4. Arance AM, Berrocal A, Lopez-Martin JA, et al. Safety of vemurafenib in patients with BRAF, V600, mutated metastatic melanoma: the Spanish experience. Clin Transl Oncol. 2016;18(11):1147.

5. Liu L, Mayes PA, Eastman S, et al. The BRAF and MEK inhibitors dabrafenib and trametinib: effects on immune function and in combination with immunomodulatory antibodies targeting PD-1, PD-L1, and CTLA-4. Clin Cancer Res. 2015;21(7):1639-1651.

6. Puzanov I. Combining targeted and immunotherapy: BRAF inhibitor dabrafenib (D) \pm the MEK inhibitor trametinib (T) in combination with ipilimumab (Ipi) for $\mathrm{V} 600 \mathrm{E} / \mathrm{K}$ mutation-positive unresectable or metastatic melanoma (MM). J Transl Med. 2015;13(1):1-2.

7. Cebollero A, Puértolas T, Pajares I, et al. Comparative safety of BRAF and MEK inhibitors (vemurafenib, dabrafenib and trametinib) in firstline therapy for BRAF-mutated metastatic melanoma. Mol Clin Oncol. 2016;5(4):458-462.

8. Queirolo P, Picasso V, Spagnolo F. Combined BRAF and MEK inhibition for the treatment of BRAF-mutated metastatic melanoma. Cancer Treat Rev. 2015;41(6):519-526.

9. Long GV, Flaherty KT, Stroyakovskiy D, et al. Dabrafenib plus trametinib versus dabrafenib monotherapy in patients with metastatic BRAF V600E/K-mutant melanoma: long-term survival and safety analysis of a phase 3 study. Ann Oncol. 2017;28(7):1631-1639.

10. Johnson DB, Flaherty KT, Weber JS, et al. Combined BRAF (dabrafenib) and MEK inhibition (trametinib) in patients with BRAFV600mutant melanoma experiencing progression with single-agent BRAF inhibitor. J Clin Oncol. 2014;32(33):3697-3704.

11. Long GV, Stroyakovskiy D, Gogas H, et al. Dabrafenib and trametinib versus dabrafenib and placebo for Val600 BRAF-mutant melanoma: a multicentre, double-blind, phase 3 randomised controlled trial. Lancet. 2015;386(9992):444-451.

12. Larkin J, Ascierto PA, Dreno B, et al. Combined vemurafenib and cobimetinib in BRAF-mutated melanoma. $N$ Engl J Med. 2014; 371(20):1867-1876.

13. Schadendorf D, Amonkar MM, Stroyakovskiy D, et al. Health-related quality of life impact in a randomised phase III study of the combination of dabrafenib and trametinib versus dabrafenib monotherapy in patients with BRAF V600 metastatic melanoma. Eur J Cancer. 2015; 51(7):833-840.

14. Robert C, Karaszewska B, Schachter J, et al. Improved overall survival in melanoma with combined dabrafenib and trametinib. N Engl J Med. 2015;372(1):30-39.
15. Corcoran RB, Atreya CE, Falchook GS, et al. Combined BRAF and MEK inhibition with dabrafenib and trametinib in BRAF V600-mutant colorectal cancer. J Clin Oncol. 2015;33(34):4023-4031.

16. Flaherty KT, Infante JR, Daud A, et al. Combined BRAF and MEK inhibition in melanoma with BRAF V600 Mutations. $N$ Engl J Med. 2012;367(18):1694-1703.

17. Patel BG, Ahmed KA, Johnstone PA, et al. Initial experience with combined BRAF and MEK inhibition with stereotactic radiosurgery for BRAF mutant melanoma brain metastases. Melanoma Res. 2016; 26(4):382-388.

18. Schmitt RJ, Kreidler SM, Glueck DH, et al. Correlation between early FDG PET/CT response to BRAF and MEK inhibition and survival in patients with BRAF-mutant metastatic melanoma. Nucl Med Commun. 2016;37(2):122-128.

19. Kim A, Cohen MS. The discovery of vemurafenib for the treatment of BRAF-mutated metastatic melanoma. Expert Opin Drug Discov. 2016;11(9):907-916.

20. Dossett LA, Kudchadkar RR, Zager JS. BRAF and MEK inhibition in melanoma. Expert Opin Drug Saf. 2015;14(4):559-570.

21. Richman J, Martinliberal J, Diem S, et al. BRAF and MEK inhibition for the treatment of advanced BRAF mutant melanoma. Expert Opin Pharmacother. 2015;16(9):1285-1297.

22. Welsh SJ, Corrie PG. Management of BRAF and MEK inhibitor toxicities in patients with metastatic melanoma. Ther Adv in Med Oncol. 2015;7(2):122-136.

23. Pinto R, Strippoli S, Summa SD, et al. MicroRNA expression in BRAF-mutated and wild-type metastatic melanoma and its correlation with response duration to BRAF inhibitors. Expert Opin Ther Targets. 2015;19(8):1027-1035.

24. Johnson AS, Crandall H, Dahlman K, et al. Preliminary results from a prospective trial of preoperative combined BRAF, and MEK-targeted therapy in advanced BRAF, mutation-positive melanoma. $J$ Am Coll Surg. 2015;220(4):581-593.

25. Galliker NA, Murer C, Kamarashev J, et al. Clinical observation of panniculitis in two patients with BRAF-mutated metastatic melanoma treated with a combination of a BRAF inhibitor and a MEK inhibitor. Eur J Dermatol. 2015;25(2):177-180.

26. Khoja L, Hogg D. Dabrafenib in the treatment of metastatic or unresectable melanoma. Expert Rev Anticancer Ther. 2015;15(3):265-276.

27. Dhillon S. Dabrafenib plus trametinib: a review in advanced melanoma with a BRAF, V600, mutation. Target Oncol. 2016;11(3):417-428.

28. Tietze MJK, Heppt M, Graf SA, et al. Aktuelle und neue Standards in der therapie. Info Onkologie. 2015;18(8):40-46.

29. Herms F, Kramkimel N, Regnierrosencher E, et al. Age and clear eyes are associated with an increased risk of cutaneous squamous cell carcinomas in vemurafenib-treated melanoma patients. Melanoma Res. 2016;26(5):487-491.

30. Schmid S, Siano M, Joerger M, et al. Response to dabrafenib after progression on vemurafenib in a patient with advanced BRAF V600E-mutant bronchial adenocarcinoma. Lung Cancer. 2015;87(1): $85-87$.
OncoTargets and Therapy

\section{Publish your work in this journal}

OncoTargets and Therapy is an international, peer-reviewed, open access journal focusing on the pathological basis of all cancers, potential targets for therapy and treatment protocols employed to improve the management of cancer patients. The journal also focuses on the impact of management programs and new therapeutic agents and protocols on

Submit your manuscript here: http://www.dovepress.com/oncotargets-and-therapy-journal
Dovepress

patient perspectives such as quality of life, adherence and satisfaction. The manuscript management system is completely online and includes a very quick and fair peer-review system, which is all easy to use. Visit http://www.dovepress.com/testimonials.php to read real quotes from published authors. 